\title{
Ranibizumab in Macular Edema Secondary to Retinal Vein Occlusion in a Real Life Practice: A Retrospective Case Series
}

\author{
Abdullah Ozkaya, Zeynep Alkin, Ceren Yesilkaya, Gurkan Erdogan, Irfan Perente, Muhittin Taskapili \\ Beyoglu Eye Training and Research Hospital, Istanbul, Turkey
}

\begin{abstract}
Objectives: The present study evaluated treatment outcomes of intravitreal ranibizumab (IVR) use in patients with macular edema (ME) secondary to retinal vein occlusion (RVO), and mean number of visits and injections of first year of treatment.

Methods: The study was a retrospective case series. Newly diagnosed RVO patients who had macular edema for $<3$ months at first admission, were treatment naïve for ME, and had follow-up of at least 12 months were included. Some patients received initial loading dose of 3 consecutive monthly injections. There were no strict criteria for administering loading dose. Patients were followed monthly, and single injection of IVR was repeated when visual acuity decreased by I or more lines on Early Treatment Diabetic Retinopathy Study chart compared to most recent visit, or any increase in central retinal thickness (CRT) in optical coherence tomography images was observed. Primary outcome measures of this study included change in best corrected visual acuity (BCVA) and CRT. Secondary outcome measures were number of visits and number of injections administered.

Results: Mean BCVA at baseline and months $3,6,9$, and 12 was $0.27 \pm 0.27$ decimals (range: $0.1-0.8$ ), $0.42 \pm 0.28$ decimals (range: $0.1-1.0$ ), $0.39 \pm 0.26$ decimals, (range: $0.01-0.8$ ), $0.37 \pm 0.29$ decimals (range: $0.01-0.9$ ), and $0.42 \pm 0.30$ decimals (range: 0.01-0.9), respectively. Mean BCVA was statistically better than mean baseline BCVA at all time points except month 9 ( $p=0.06$ for month 9, and $p<0.05$ for month 3, 6, and 12). Mean CRT at baseline, months 3, 6, 9, and I2 was

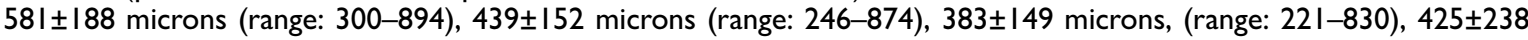
microns (range: 235-I 147), and 359 $\pm 10 \mathrm{I}$ microns (range: 229-655), respectively. Mean CRT level was statistically lower than mean baseline BCVA ( $p<0.05$ for all) at all time points. At month 12, 17 of the 45 patients $(37.8 \%)$ had anatomically inactive ME and did not require injections. Mean number of planned visits at month 12 was $4.8 \pm 1.0$ (range: 2-7), and number of completed visits was $4.5 \pm$ I.2 (range: I-6) (94.2\% completion). Mean number of planned injections at month 12

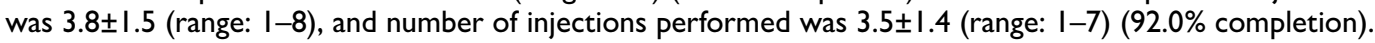

Conclusion: Ranibizumab is an effective agent in treatment of ME secondary to RVO with regard to visual and anatomical outcomes. Number of visits and injections were lower than prospective multicenter studies, as expected, yet functional and anatomical outcomes were acceptable.
\end{abstract}

Keywords: Macular edema, ranibizumab, retinal vein occlusion.

\section{Introduction}

Retinal vein occlusion (RVO) is the second most common type of retinal vascular disorder after diabetic retinopathy, and macular edema (ME) and vitreous hemorrhage are fre- quent causes of visual loss in patients with RVO (I-5). Inflammation and increased vascular endothelial growth factor (VEGF) levels both play important role in pathogenesis of ME secondary to RVO (4-7). Several treatment options such

Address for correspondence: Abdullah Ozkaya, MD. Beyoglu Eye Training and Research Hospital, Bereketzade Cami Sok., 34421 Beyoglu, Istanbul, Turkey

Phone: +90 21225159 00, E-mail: abdozkaya@gmail.com

Submitted Date: October 17, 2016 Accepted Date: November 05, 2016 Available Online Date: November 15, 2016 ${ }^{\circ}$ Copyright 2016 by Beyoglu Eye Training and Research Hospital - Available online at www.beyoglueye.com 
as laser photocoagulation, vitreoretinal surgery, intravitreal anti-VEGF and steroid injections, as well as various surgical techniques have been reported to be effective in treatment of ME secondary to RVO (I-8). Currently, intravitreal injections of anti-VEGF agents or steroids are preferred as first line treatment option for ME (8). Bevacizumab, ranibizumab, and aflibercept are 3 anti-VEGF agents used; the first is off-label, while the other 2 have been approved for treatment of ME $(2,8,9-12)$. In pivotal multicenter studies with strict follow-up and treatment criteria, successful treatment outcomes have been reported (9-13). However, it is usually not possible to follow these strict criteria in real-life practice (14-16). Number of injections, in particular, has been found to be very low in real-life studies in comparison to multicenter studies (14-16). In the present study, we aimed to evaluate outcomes of intravitreal ranibizumab (IVR) treatment in patients with ME secondary to RVO, as well as mean number of visits and injections during first year of treatment.

\section{Methods}

In this retrospective case series, medical records of patients who had ME secondary to RVO and who underwent IVR treatment between January and December of 2014 were reviewed. Newly diagnosed RVO patients who had macular edema $<3$ months on first admission, were treatment naïve for $M E$, and had follow-up of at least 12 months were included. Patients who had co-existing retinal disease (such as diabetic retinopathy or epiretinal membrane), or media opacities that could decrease visual acuity (VA) were not included. Written informed consent for treatment was obtained from all patients, and the study adhered to tenets of the Declaration of Helsinki.

Data collected from patients' records included age, gender, type of RVO, ischemic status, best corrected visual acuity (BCVA), and central retinal thickness (CRT) at baseline and months 3,6, 9, and 12 , as well as number of visits and number of injections.

All patients underwent standardized examination including measurement of BCVA using Early Treatment Diabetic Retinopathy Study (ETDRS) chart at 4 meters, slit-lamp biomicroscopy and fundus examination, and measurement of intraocular pressure via applanation tonometry. Fundus photography, fluorescein angiography (HRA-2; Heidelberg Engineering, Heidelberg, Germany), and optical coherence tomography (OCT) imaging (Spectralis; Heidelberg Engineering, Heidelberg, Germany) were performed before treatment. All examinations were repeated at all visits except fluorescein angiography, which was repeated only when cause of VA deterioration could not be clarified with clinical examination and other imaging methods. OCT was used to measure CRT, which was defined as mean thickness of the neurosensory retina in central $\mathrm{I} \mathrm{mm}$ diameter region, and was computed via OCT mapping software provided with device. Fluorescein angiography results were examined for capillary dropout zones at the fovea and peripheral retina, and for leakage, which is accepted cause of ME. Type of disease was defined as ischemic RVO if ischemic area was $\geq 5$ disc areas in branch retinal vein occlusion (BRVO) patients, or $\geq 10$ disc areas in central retinal vein occlusion (CRVO) patients.

All injections were performed under sterile conditions after application of topical anesthesia, use of $10 \%$ povidone-iodine (Betadine; Purdue Pharma, Stamford, CT, USA) scrub on eyelids and eyelashes, and $5 \%$ povidone-iodine on conjunctival sac. IVR $0.5 \mathrm{mg} / 0.5 \mathrm{~mL}$ (Lucentis; Novartis Pharma, Basel, Switzerland) was injected through the pars plana at 3.5 $\mathrm{mm}$ posterior to the limbus with 30-gauge needle. Patients were instructed to return to the hospital if they experienced decreased vision, eye pain, or any new symptoms.

Some patients received initial loading dose of 3 consecutive monthly injections. There were no strict criteria for administration of loading dose. Patients were followed monthly, and single injection of IVR was repeated when VA decreased by I or more lines on ETDRS chart compared to most recent visit, or any increase in CRT was seen in OCT images.

Primary outcome measures of this study included change in BCVA and CRT. Secondary outcome measures were number of visits and number of injections.

\section{Statistical Analysis}

VA was converted to logarithm of minimum angle of resolution (LogMAR) for statistical analysis. Categorical variables were presented as numbers and percentages, while numerical variables were expressed as mean and standard deviation. First, data were analyzed in terms of normality using Shapiro-Wilk test. As distribution of the data was found to be normal, VA and CRT values between baseline and other time points were assessed with repeated measures test. Categorical variables were compared using chi-square test. A p value $<0.05$ was considered statistically significant.

\section{Results}

Forty-five eyes of 45 patients were included in the study. Baseline general characteristics were summarized in Table I.

Mean BCVA at baseline and months 3, 6, 9, and 12 was $0.27 \pm 0.27$ decimals (range: $0.1-0.8$ ), $0.42 \pm 0.28$ decimals (range: $0.1-1.0$ ), $0.39 \pm 0.26$ decimals, (range: $0.01-0.8$ ), $0.37 \pm 0.29$ decimals (range: $0.0 \mathrm{I}-0.9$ ), and $0.42 \pm 0.30$ decimals (range: $0.0 \mathrm{I}-0.9$ ), respectively (Figure I, Table 2). With exception of month 9, mean BCVA was statistically better at all time points than mean baseline BCVA $(p=0.01$ for month 
Table I. General characteristics of the patients

Number of eyes

45

Age (years)

Gender (male/female)

$56.6 \pm 11.7$

Hypertension (\%)

$27 / 18$

Diabetes (\%)

$34(75.5 \%)$

Hyperlipidemia (\%)

II (24.4\%)

$3(6.6 \%)$

Fluoroscein Angiography (non-ischemic/ischemic)

$18 / 9$

Type of RVO (BRVO/CRVO)

$33 / 12$

Lens status (phakic/pseudophakic)

$37 / 8$

Baseline BCVA (in decimals)

$0.27 \pm 0.27$

Baseline CRT (microns)

$58 \mathrm{I} \pm 188$

BCVA: best corrected visual acuity; BRVO: branch retinal vein occlusion; CRT: central retinal thickness; CRVO: central retinal vein occlusion; RVO: retinal vein occlusion.

3, $p<0.000$ I for month $6, p=0.06$ for month $9,0=0.005$ for month 12). Sixteen (35.5\%) of the 45 patients had gained $\geq$ 3 LogMAR lines of VA at month 12. Percentage of patients who had stable visual acuity (lost $<3$ lines, stable, or gained $<3$ lines) at month 12 was $57.7 \%$ (26/45), and only 3 patients $(6.6 \%)$ lost $\geq 3$ lines of VA.

Mean CRT at baseline and months 3, 6, 9, and 12 was $58 \mathrm{I} \pm 188$ microns (range: 300-894), 439 152 microns (range: 246-874), 383 \pm 149 microns (range: 22I-830), $425 \pm 238$ microns (range: $235-1147$ ), and $359 \pm 101$ microns (range: 229-655), respectively (Figure 2, Table 2). Mean CRT level was statistically lower than mean baseline BCVA at all time points $(p=0.01$ for month $3, p<0.0001$ for month 6 , $p=0.001$ for month $9, p<0.000$ I for month 12). At month I2, 17 of the 45 patients (37.8\%) had anatomically inactive ME and did not require injections.

Mean number of planned visits at month 12 was $4.8 \pm 1.0$ (range: $2-7$ ), and number of completed visits was $4.5 \pm 1.2$ (range: 1-6) (94.2\% completion). Mean number of planned injections at month 12 was $3.8 \pm 1.5$ (range: $1-8$ ), and the number of injections performed was $3.5 \pm 1.4$ (range: I-7) (92.0\% completion). Twenty-six patients $(57.8 \%)$ received loading dose of 3 consecutive monthly injections.

No injection-related endophthalmitis was noted after to-

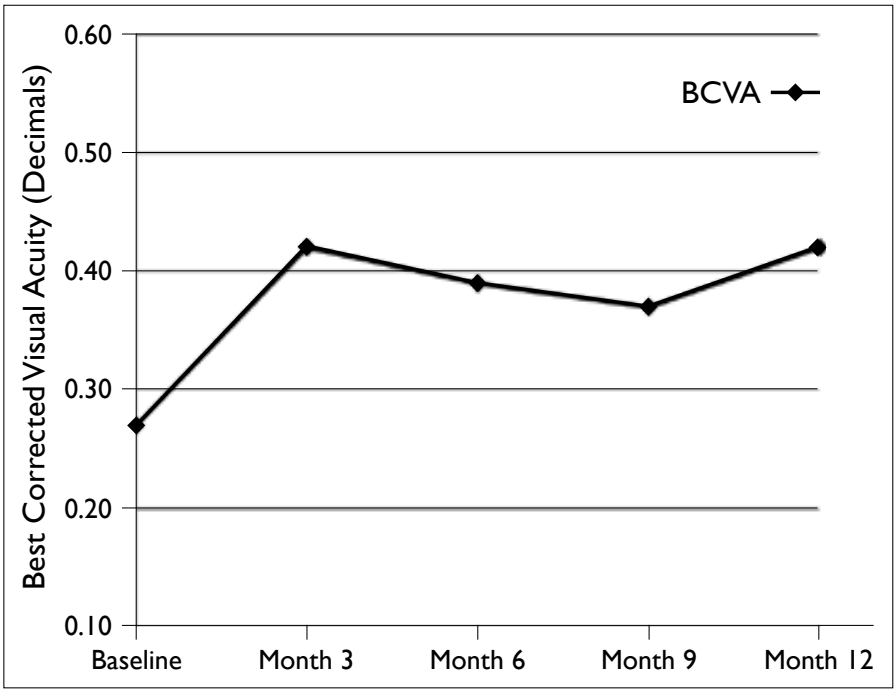

Figure I. The graph shows change in mean visual acuity levels from baseline to month 12 .

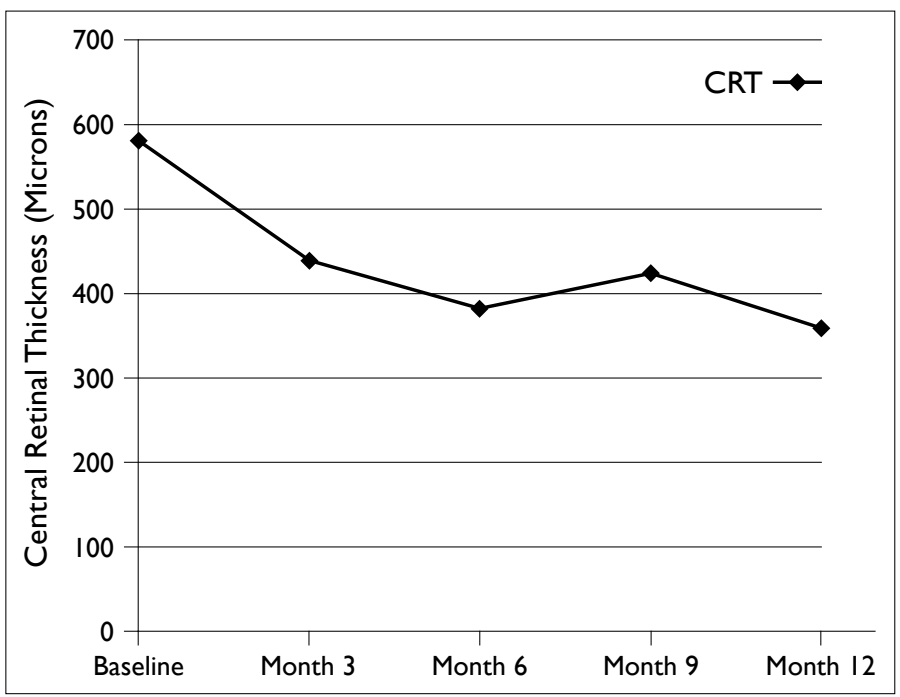

Figure 2. The graph shows change in central retinal thickness from baseline to month 12 .

tal of 161 injections.

\section{Discussion}

In this study, 12 months of real-life outcomes of IVR treatment for ME secondary to RVO were evaluated. Baseline visual acuity increased significantly from 0.27 to 0.42 deci-

Table 2. Mean best corrected visual acuity and central retinal thickness levels at different time points

\begin{tabular}{lccccc} 
& Baseline & Month 3 & Month 6 & Month 9 & Month I2 \\
BCVA, in decimal & $0.27 \pm 0.27$ & $0.42 \pm 0.28$ & $0.39 \pm 0.26$ & $0.37 \pm 0.29$ & $0.42 \pm 0.30$ \\
$($ LogMAR) & $(0.85 \pm 0.6 I)$ & $(0.47 \pm 0.33)$ & $(0.58 \pm 0.5 I)$ & $(0.73 \pm 0.7 \mathrm{I})$ & $(0.57 \pm 0.5 \mathrm{I})$ \\
CRT, microns & $581 \pm 188$ & $439 \pm 152$ & $383 \pm 149$ & $425 \pm 238$ & $359 \pm 101$ \\
\hline
\end{tabular}

BCVA: best corrected visual acuity; CRT: central retinal thickness; LogMAR: logarithm of minimum angle of resolution. 
mals as early as month 3 and remained significantly better through month I2. CRT level was also found to be significantly decreased at month 3 and remained significantly better at months 6, 9, and 12. Mean visit and injection numbers were lower than multicenter studies $(9-13,17)$, but were similar to other real-life studies (14-16).

In multicenter, prospective studies it is possible to achieve proper follow-up schedule $(9-13,17)$ and adhere to tight injection criteria. Such circumstances yield good visual and anatomical outcomes. In pivotal studies of ranibizumab, 6 consecutive monthly injections were performed with additional 6 months of follow-up and treatment as needed. In the Branch Retinal Vein Occlusion (BRAVO) study, 18 letters of visual increase and 347 microns decrease in CRT were reported after 12 months, with mean of 8.5 injections (17). In the Central Retinal Vein Occlusion (CRVO) study, known as the CRUISE study, the same treatment regimen was applied to CRVO patients. At month I2, VA increased by 13.9 letters, and CRT had decreased by 462 microns with mean of 8.8 injections (17). Very successful visual and anatomical outcomes were also reported in some other single-center, prospective studies (18). Chang et al. evaluated CRVO patients who were treated with ranibizumab and had mean follow-up of 12 months (18). Patients received 3 monthly IVR injections followed by pro re nata regimen. At month 12, mean visual acuity was found to have increased by 17.8 letters and CRT had decreased by 263 microns with mean of 10.2 injections.

On the other hand, it is very difficult to adhere to strict follow-up and treatment criteria in real-life practice $(14,16)$. In several important studies of anti-VEGF drugs in which ME secondary to RVO was analyzed, 6 initial monthly injections were performed (9-12). Varied treatment regimens were subsequently applied (9-13). Loading phase of anti-VEGF drugs was questioned in some studies, and less frequent ranibizumab treatment was found to be effective (I5). Fewer follow-up visits and injections in real-life practice usually led to decrease in visual and anatomical outcomes in patients with diabetic macular edema (DME) and macular degeneration (nAMD) $(18,19)$. However, this is not the case for ME secondary to RVO, which is not usually chronic disease like DME and nAMD $(4-6,20)$. In a prospective study conducted by Miwa et al., $8 \mathrm{I}$ eyes with ME secondary to BRVO were evaluated (I5). Some patients received 3 initial monthly IVR injections while second group received only I injection prior to treatment as needed for remainder of 12-month study period. At the conclusion of follow-up, the 2 groups had similar results in terms of visual outcomes. In another study, Skanishi et al. evaluated IVR treatment for ME secondary to RVO and at conclusion of follow-up period of 12 months. Patients in BRVO group received mean of 2.1 injections, and 3.4 injections were administered in CRVO group, very small number of doses in comparison to multicenter studies (16). Despite few injections, VA change from baseline to month 6 was reported to be 2.8 LogMAR lines and 2.5 lines at month 12 in CRVO group, and 1.8 lines at month 6, and 2.1 lines at month 12 in BRVO group, which were comparable outcomes to aforementioned studies (9-13, 16). In present study, BRVO and CRVO patients were evaluated together and similar results were seen at month 12 .

The main limitation of this study was its retrospective design and relatively low number of patients. However, the study included only treatment naïve patients and revealed some useful data for real life. Perhaps the most valuable data were visit and injection completion rates. The main problem with anti-VEGF treatment in real life is frequency of visits and injections (19). Usually, physicians try to call patients for monthly visits, or at least believe that they call patients monthly. However, in our study, both visit and injection frequencies were very far from ideal. One other problem in real life can be patient compliance, yet our study demonstrated visit completion rate of $94.2 \%$ and injection completion rate of $92.0 \%$, which were quite acceptable rates for patient compliance. Therefore, upon analysis of results of this study, we realized drawback of our patient follow-up and re-treatment criteria for this group of ME secondary to RVO patients. The compliance of the patients was quite good, yet visit and injection numbers were very low. We are now trying to conduct more frequent follow-up visits and we hope that increased number of visits will lead to increased number of injections. After re-organizing our clinic we will conduct another study to evaluate our progress in that regard.

In conclusion, ranibizumab is an effective agent in treatment of ME secondary to RVO with respect to visual and anatomical outcomes. Number of visits and injections was lower than prospective, multicenter studies, as expected, but functional and anatomical outcomes were acceptable.

\section{Disclosures}

Financial Disclosure: This retrospective study was not supported by any company. None of the authors has financial or proprietary interests in any material or method mentioned. These data have not been previously published.

Peer-review: Externally peer-reviewed.

Conflict of Interest: None declared.

Authorship Contributions: Involved in design and conduct of the study (AO, ZTA, CY, GE, IP, MT); preparation and review of the study (AO, ZTA, GE, MT); data collection (AO, CY, IP); and statistical analysis $(A O)$.

\section{References}

I. Scott IU, VanVeldhuisen PC, Oden NL, IP MS, Blodi BA, Jumper JM, et al. SCORE Study report I: baseline associations between central retinal thickness and visual acuity in patients with retinal 
vein occlusion. Ophthalmology 2009; I 16:504-12. Crossre

2. Donati S, Barosi P, Bianchi M, Al Oum M, Azzolini C. Combined intravitreal bevacizumab and grid laser photocoagulation for macular edema secondary to branch retinal vein occlusion. Eur J Ophthalmol 2012;22:607-14. Crossre

3. Argon laser photocoagulation for macular edema in branch vein occlusion. The Branch Vein Occlusion Study Group. Am J Ophthalmol 1984;98:271-82. Crossre

4. Augustin AJ, Sahel JA, Cerulli L, Texier-Richard B, Buchholz PM, Kobelt $\mathrm{G}$. Treating retinal vein occlusions in France, Germany, and Italy: an analysis of treatment patterns, resource consumption, and costs. Eur J Ophthalmol 2012;22:776-84 Crossre

5. Bhagat N, Goldberg MF, Gascon P, Bell W, Haberman J, Zarbin MA. Central retinal vein occlusion: review of management. Eur J Ophthalmol 1999;9:165-80.

6. Glacet-Bernard A, Coscas G, Zourdani A, Soubrane G, Souied $E H$. Steroids and macular edema from retinal vein occlusion. Eur J Ophthalmol 201 I;21 Suppl 6:37-44. Crossre

7. Noma H, Funatsu H, Mimura T, Hori S. Changes of vascular endothelial growth factor after vitrectomy for macular edema secondary to retinal vein occlusion. Eur J Ophthalmol 2008; 18:1017-9.

8. Yumusak E, Buyuktortop N, Ornek K. Early results of dexamethasone implant, ranibizumab, and triamcinolone in macular edema due to branch retinal vein occlusion. Eur J Ophthalmol 2016;26:54-9. Crossre

9. Brown DM, Campochiaro PA, Bhisitkul RB, Ho AC, Gray S, Saroj N, et al. Sustained benefits from ranibizumab for macular edema following branch retinal vein occlusion: 12-month outcomes of a phase III study. Ophthalmology 201 I; I 18:1594-602

10. Brown DM, Campochiaro PA, Singh RP, Li Z, Gray S, Saroj N, et al. Ranibizumab for macular edema following central retinal vein occlusion: six-month primary end point results of a phase III study. Ophthalmology 2010; I 17: I 124-33. Crossre

Il. Ogura Y, Roider J, Korobelnik JF, Holz FG, Simader C, Schmidt-Erfurth $U$, et al. Intravitreal aflibercept for macular edema secondary to central retinal vein occlusion: 18-month results of the phase 3 GALILEO study. Am J Ophthalmol 20।4; I58:1032-8. Crossre
I2. Brown DM, Heier JS, Clark WL, Boyer DS, Vitti R, Berliner AJ, et al. Intravitreal aflibercept injection for macular edema secondary to central retinal vein occlusion: I-year results from the phase 3 COPERNICUS study. Am J Ophthalmol 20I 3; 155:429_ 37. Crossret

I3. Clark WL, Boyer DS, Heier JS, Brown DM, Haller JA, Vitti R, et al. Intravitreal Aflibercept for Macular Edema Following Branch Retinal Vein Occlusion: 52-Week Results of the VIBRANT Study. Ophthalmology 2016;123:330-6. Crossre

14. Ozkaya A, Celik U, Alkin Z, Faiz Turan M, Yazici AT, Demirok A. Comparison between Intravitreal Triamcinolone with Grid Laser Photocoagulation versus Bevacizumab with Grid Laser Photocoagulation Combinations for Branch Retinal Vein Occlusion. ISRN Ophthalmol 2013;20 I3:14I 279.

15. Miwa Y, Muraoka Y, Osaka R, Ooto S, Murakami T, Suzuma $\mathrm{K}$, et al. Ranıbızumab for macular edema after branch retınal vein occlusıon: One Initial Injection Versus Three Monthly Injections. Retina 2016. Crossre

16. Sakanishi Y, Lee A, Usui-Ouchi A, Ito R, Ebihara N. Twelvemonth outcomes in patients with retinal vein occlusion treated with low-frequency intravitreal ranibizumab. Clin Ophthalmol 2016;10:1161-5. Crossre

17. Thach $A B$, Yau L, Hoang $C$, Tuomi L. Time to clinically significant visual acuity gains after ranibizumab treatment for retinal vein occlusion: BRAVO and CRUISE trials. Ophthalmology 2014;121:1059-66. Crossre

18. Chang LK, Spaide RF, Klancnik JM, Sorenson J, Slakter JS, Freund $\mathrm{KB}$, et al. Longer-term outcomes of a prospective study of intravitreal ranibizumab as a treatment for decreased visual acuity secondary to central retinal vein occlusion. Retina 201 I;31:821-8. Crossre

19. Holz FG, Tadayoni R, Beatty S, Berger AR, Cereda MG, Hykin P, et al. Determinants of visual acuity outcomes in eyes with neovascular AMD treated with anti-VEGF agents: an instrumental variable analysis of the AURA study. Eye (Lond) 2016;30:1063-7I.

20. Argon laser scatter photocoagulation for prevention of neovascularization and vitreous hemorrhage in branch vein occlusion. A randomized clinical trial. Branch Vein Occlusion Study Group. Arch Ophthalmol 1986;104:34-4I. Crossre 